
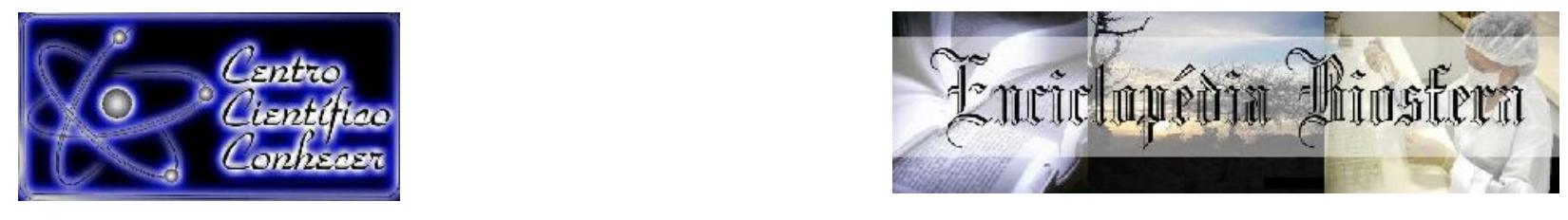

\title{
OSTEOSSARCOMA EM ESCÁPULA COM METÁSTASE CUTÂNEA EM CADELA DA RAÇA POODLE - RELATO DE CASO
}

Guilherme Henrique Salazar Badan ${ }^{1}$, Fernanda Maria Ozelim de Pádua Alamy Martins $^{2}$, Marcelo Seixo de Brito e Silva ${ }^{3}$, Eugênio Gonçalves de Araújo ${ }^{4}$, Vanessa de Sousa Cruz ${ }^{5}$

${ }^{1}$ Graduando em Medicina Veterinária da Escola de Veterinária e Zootecnia da Universidade Federal de Goiás - Brasil.

${ }^{2}$ Médica Veterinária da Seção de Cães de Guerra do Batalhão da Polícia do Exército de Brasília - Brasil

${ }^{3}$ Professor Doutor do Instituto de Ciências Biológicas da Universidade Federal de Goiás - Brasil

${ }^{4}$ Professor Doutor do Setor de Patologia Veterinária da Universidade Federal de Goiás - Brasil

${ }^{5}$ Pós doutoranda PNPD - Laboratório Multiusuário de Avaliação de Moléculas, Células e Tecidos da Universidade Federal de Goiás - Brasil e-mail de contato:salazarbadan@gmail.com

Recebido em: 06/04/2019 - Aprovado em: 10/06/2019 - Publicado em: 30/06/2019 DOI: 10.18677/EnciBio_2019A118

O osteossarcoma é a principal neoplasia óssea maligna nos cães. Corresponde a $6 \%$ das neoplasias incidentes na espécie e está associado a prognóstico reservado. Acomete, principalmente, os ossos longos que suportam o peso do esqueleto apendicular, nas raças de porte grande e gigante. Pode ocorrer, também, no esqueleto axial, assim como em tecidos moles, no entanto, com menor frequência. Esse artigo relata o caso de uma cadela Poodle, de 12 anos de idade, com osteossarcoma osteoblástico na escápula direita, seguido de metástase cutânea na face interna da raiz do membro pélvico esquerdo.

PALAVRAS-CHAVE: câncer, oncologia veterinária, tumor ósseo.

\section{CASE REPORT: SCAPULAR OSTEOSARCOMA WITH CUTANEOUS METASTASIS ON FEMALE POODLE DOG}

\begin{abstract}
Osteosarcoma is the main malignant bone neoplasm in dogs. Corresponds to $6 \%$ of neoplasms incident to the species and is associated with reserved prognosis. It mainly affects the weight bearing long bones of the appendicular skeleton, in the races of large and giant size. It can also occur in the axial skeleton as well as in soft tissues, however, less frequently. This article reports the case of a 12 year old Poodle female dog with osteoblastic osteosarcoma in the right scapula, followed by cutaneous metastasis on the inner face of the left pelvic limb.
\end{abstract}

KEYWORDS: cancer, veterinary oncology, bone tumor. 


\section{INTRODUÇÃO}

O osteossarcoma é uma neoplasia óssea maligna que acomete tanto humanos quanto animais. Em cães, esse tipo de neoplasia pode acontecer tanto no esqueleto axial, quanto no esqueleto apendicular, porém, cerca de $75 \%$ dos casos ocorrem no esqueleto apendicular. Há preferência pelos ossos longos que suportam peso, como o úmero, fêmur e tíbia (FENGER et al., 2014).

A epidemiologia para essa neoplasia ainda não está bem estabelecida, no entanto existem fatores predisponentes. Raças de porte grande e gigante, como wolfhound irlandês, deerhound escocês, dogue alemão, mastim inglês, são bernardo, golden retriever e rottweiler possuem uma pré disposição (SCHIFFMAN et al., 2015). Alguns autores relatam que machos são mais afetados do que fêmeas (SIMPSOM et al., 2017), no entanto já existem estudos que mostram que essa diferença pode não existir (SAPIERZYŃSKI; CZOPOWICZ, 2017).

O diagnóstico pode ser feito com base na apresentação clínica e técnicas de diagnóstico por imagem para um diagnóstico presuntivo. Em radiografias, as lesões podem se apresentar como líticas, mistas ou produtivas. A imagem radiográfica é dinâmica e está sujeita a mudanças, geralmente se apresentando como um padrão de explosão solar ou em alguns casos como o triângulo de Codman. Outras técnicas de diagnóstico como a tomografia computadorizada ou a ressonância magnética também podem ser utilizadas, no entanto o diagnóstico definitivo deve ser dado pelo exame histopatológico (FARCAS et al., 2014).

O osteossarcoma é caracterizado pela presença de células neoplásicas primitivas com a capacidade de produzir osteoide (SIMPSOM et al., 2017). A origem precisa dessas células ainda não é conhecida. Assume-se que tenham origem nos osteoblastos, no entanto há evidências que possam originar de osteócitos ou outras células (SOTTNIK et al., 2014; FENGER et al., 2014). Pela histologia, o osteossarcoma pode ser dividido em central, com as subdivisões indiferenciado, osteoblástico, condroblástico, telangectástico, e de células gigantes; e periférico, com as subdivisões periosteal e parosteal (SIMPSOM et al., 2017).

A primeira escolha para tratamento do osteossarcoma é a amputação cirúrgica do membro afetado (SZEWCZYK et al., 2015). Adicionalmente à cirurgia, um tratamento quimioterápico pode ser utilizado para melhorar a sobrevida dos pacientes. Em cães, a principal escolha está no uso da doxorrubicina associada à cisplatina ou carboplatina (SIMPSOM et al., 2017). Uma última opção de tratamento está no uso da radioterapia que, no entanto, não é de fácil acesso para uso veterinário (SZEWCZYK et al., 2015).

O prognóstico para o osteossarcoma é geralmente desfavorável, e mesmo com o animal sobrevivendo ao primeiro ano, o tempo de sobrevida após esse período fica em torno de oito meses (CULP et al., 2014). Em um estudo de meta análise, foram destacados três fatores principais dentre eles a Fosfatase Alcalina, em especial a Fosfatase Alcalina Óssea, por estar relacionada a atividade osteoblástica, a localização, tumores com localização no úmero proximal tendem a ser mais agressivas, e idade, no qual esse tipo de neoplasia é mais frequentemente observada em pacientes mais velhos (BOERMAN et al., 2012). 


\section{RELATO DE CASO}

Uma cadela da raça poodle, com 12 anos de idade e $9 \mathrm{~kg}$ foi encaminhada ao Setor de Patologia Animal da EVZ/UFG para realização de biópsia aspirativa para citologia. Apresentava histórico de aumento de volume na porção distal da escápula direita (Figura 1), com tempo de evolução de, aproximadamente, 60 dias. Havia claudicação e sensibilidade dolorosa à palpação. Ademais, o animal já havia passado por um exame radiológico, cujo resultado era sugestivo de neoplasia óssea.

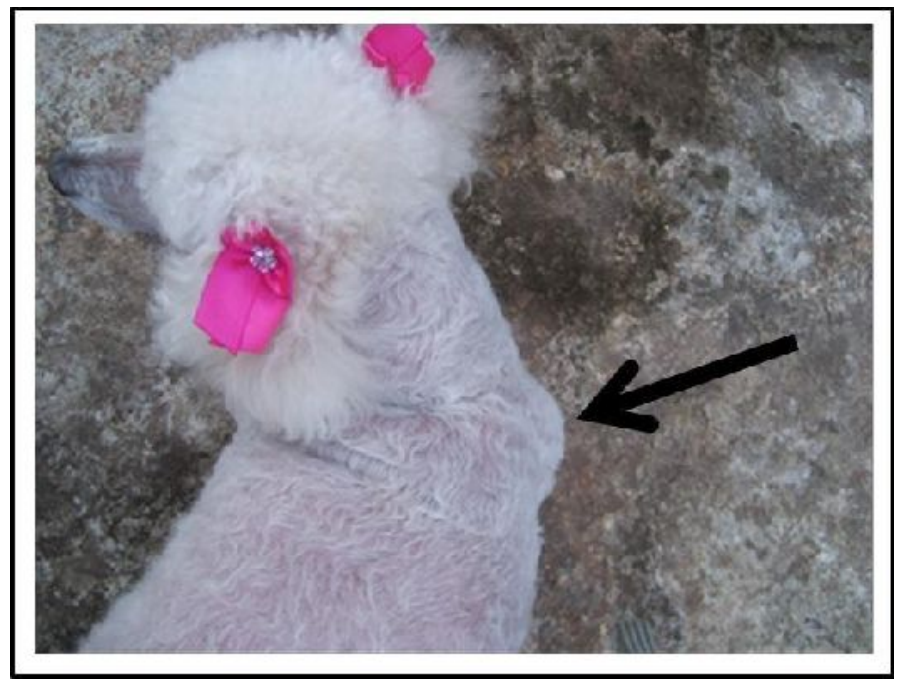

FIGURA 1 - Cadela da raça poodle, com 12 anos de idade, $9 \mathrm{~kg}$ e aumento de volume na porção distal da escápula direita.

Durante a palpação foi constatado que 0 nódulo era único, com aproximadamente $8 \mathrm{~cm}$ de diâmetro, consistência firme, não ulcerado. A citologia aspirativa por agulha fina foi feita após localização, fixação e assepsia do nódulo com álcool a $70 \%$. A agulha $(30 \times 8 \mathrm{~mm})$ foi introduzida na lesão, com movimentos repetidos em várias direções e o material colhido foi aspergido em lâminas histológicas. O esfregaço foi obtido por meio do deslizamento de outra lâmina histológica, inclinada em angulação de 45․ O material foi fixado em álcool metílico, corado com solução de Giemsa e analisado em microscópio ótico. Os achados citológicos indicaram osteossarcoma moderadamente indiferenciado.

$\mathrm{O}$ animal foi encaminhado para realização de exames pré-operatórios. A bioquímica sérica evidenciou Fosfatase Alcalina de 1369,36 UI/L, sendo o valor referencial de até $100 \mathrm{UI} / \mathrm{L}$. Os demais exames laboratoriais e radiológicos não apresentaram alterações. A paciente foi submetida à cirurgia para amputação de todo o membro anterior direito no Hospital Veterinário da EVZ/UFG. 


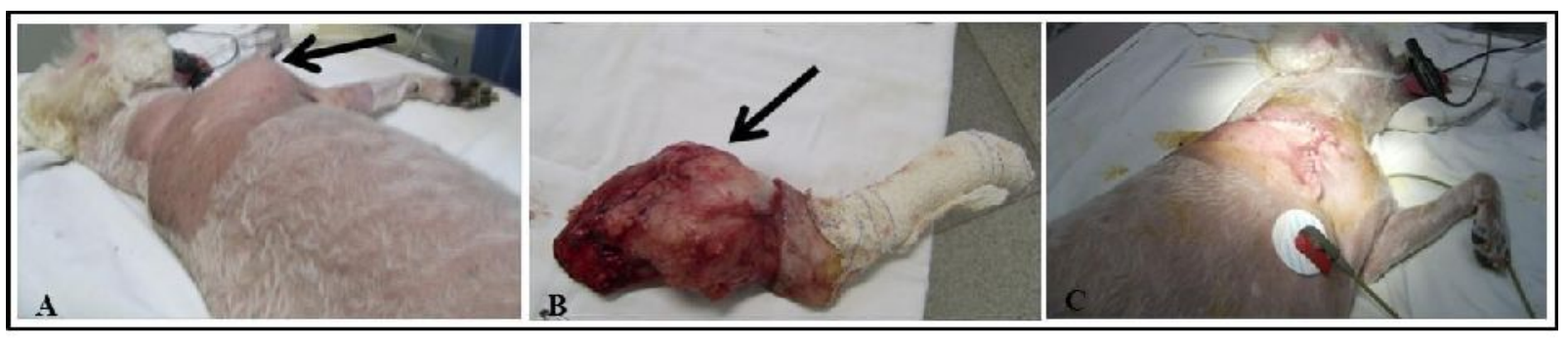

FIGURA 2 - Cadela da raça poodle. A) Paciente antes do procedimento cirúrgico com destaque para o nódulo (seta). B) Membro após a remoção com destaque para o nódulo na porção distal da escápula direita (seta). C) Aspecto da ferida cirúrgica imediatamente após o procedimento cirúrgico.

O nódulo foi conduzido ao Setor de Patologia Animal da EVZ/UFG para realização do exame histopatológico. Durante o exame histopatológico observou-se células blásticas infiltrativas, com citoplasma acidófilo e nucléolos evidentes, apoiadas sobre finas bainhas de conjuntivo. B) Restos de matriz óssea invadida pelas células tumorais. As características notadas são compatíveis com o diagnóstico de osteossarcoma osteoblástico (Figura 3).

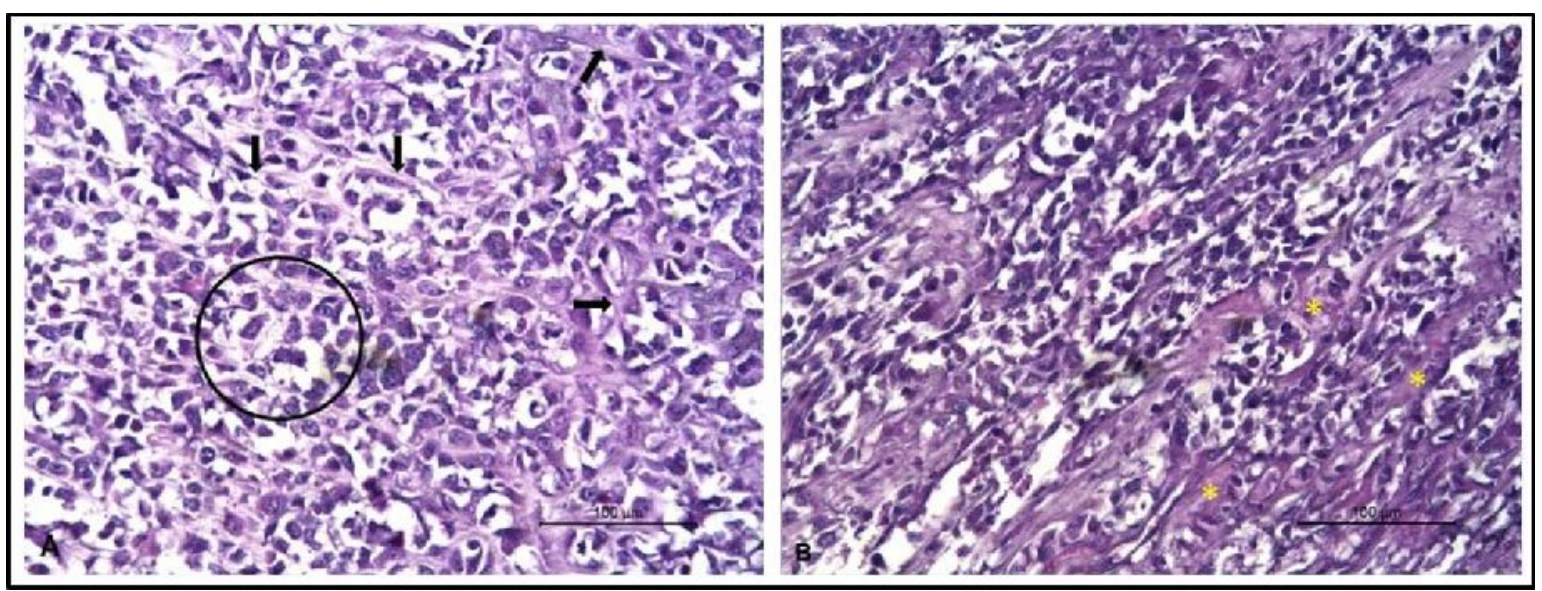

FIGURA 3 - Fotomicrografia de fragmento de osteossarcoma osteoblástico em cadela da raça poodle, localizado na porção distal da escápula direita. A) Células blásticas infiltrativas (círculo), com citoplasma acidófilo e nucléolos evidentes, apoiadas sobre finas bainhas de conjuntivo (setas). B) Restos de matriz óssea (asteriscos) invadida pelas células tumorais. H\&E.

Houve a sugestão do tratamento quimioterápico com protocolo de utilização da doxorrubicina, no entanto, a opção foi descartada pelo tutor da paciente. Após dois meses, um nódulo surgiu na face interna da raiz do membro pélvico esquerdo da paciente (Figura 4). Foi realizado um exame ultrassonográfico da estrutura, que evidenciou uma massa ecogênica, geradora de forte sombra acústica posterior, medindo $3.54 \mathrm{~cm}$ em seu maior diâmetro. Demais órgãos abdominais tópicos, apresentando ecotextura e volumes normais. A conclusão do laudo ecográfico mostrou tratar-se de uma massa sólida, calcificada, em partes moles do membro. Além disso, a radiografia torácica diagnosticou áreas de metástase pulmonar. 


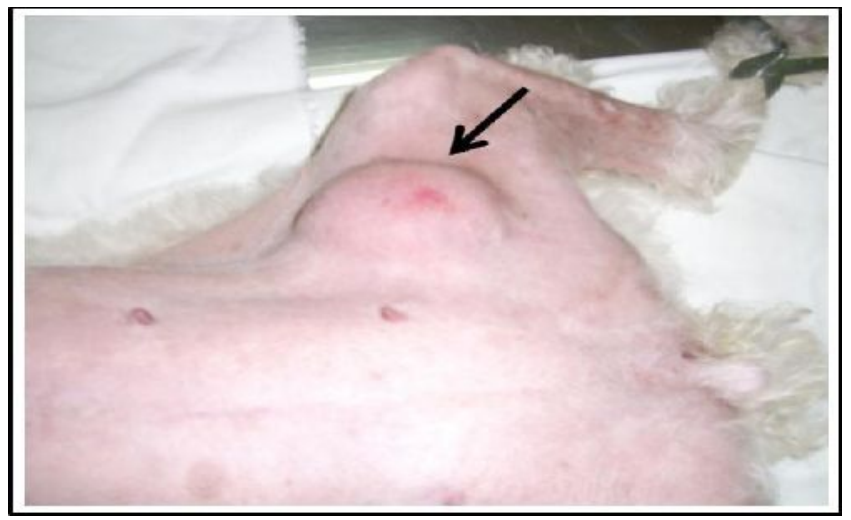

FIGURA 4 - Cadela da raça poodle com nódulo na face interna da raiz do membro pélvico esquerdo.

O cão foi encaminhado novamente à cirurgia para retirada cirúrgica do crescimento. O material foi remetido ao Laboratório de Patologia da EVZ/UFG para exame histopatológico. Observou-se proliferação de células osteoblásticas anaplásicas (setas), infiltrando-se na matriz óssea (setas) $O$ resultado mostrou tratar-se de osteossarcoma osteoblástico (Figura 5).

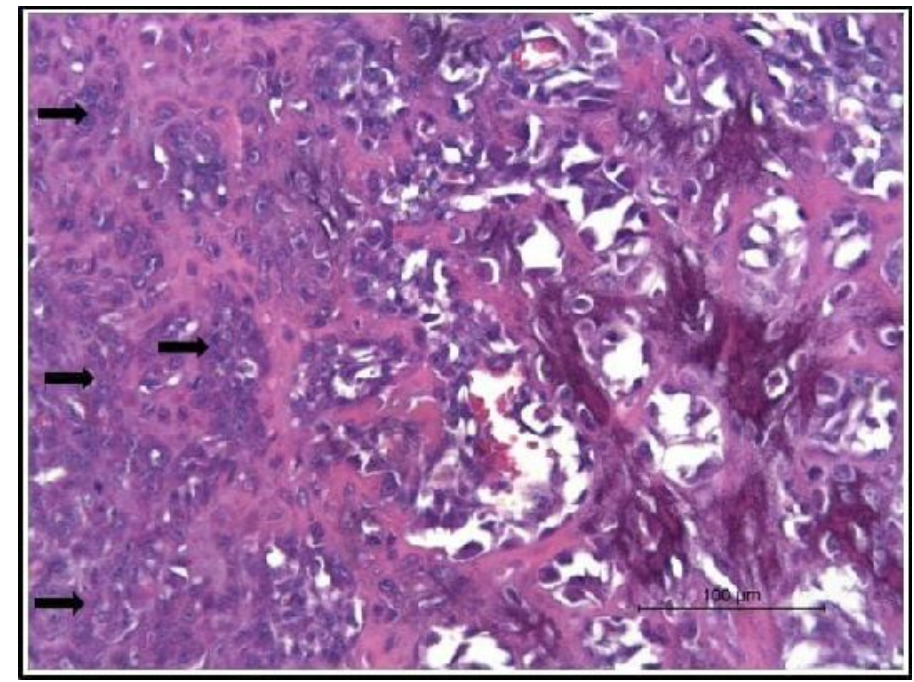

FIGURA 5 - Fotomicrografia de fragmento de osteossarcoma osteoblástico, localizado na face interna da raiz do membro pélvico esquerdo, em cadela da raça poodle. Proliferação de células osteoblásticas anaplásicas (setas), infiltrando-se na matriz óssea (setas). H\&E.

O animal veio a óbito três semanas após a segunda cirurgia.

\section{DISCUSSÃO}

No caso relatado a cadela era da raça poodle. A literatura disponível para o osteossarcoma em cães da raça Poodle ainda é bastante limitada, o que dificulta a obtenção de dados com relação ao acometimento da raça a essa neoplasia e pode 
ser compatível com a hipótese de pequena probabilidade de ocorrência. A idade de 12 anos se encontra acima da idade média de 8,2 anos reportada por Culp et al., (2014), embora também tenham relatado casos com idades variando de 2,7 a 13,3 anos. O peso de $9 \mathrm{Kg}$ está bem abaixo do peso médio relatado por Montinaro et al., (2013) de $34,8 \mathrm{Kg}$.

A cadela em estudo apresentou a lesão na porção distal da escápula direita. O acometimento de escápula por osteossarcoma é bastante raro, com a maior parte dos casos ocorrendo em esqueleto apendicular (FAN; KHANNA, 2015). De acordo com Kruse et al., (2013), a presença do osteossarcoma em escápula indica uma probabilidade 2,8 vezes maior de morte do que em outras localidades.

O exame sorológico realizado para verificar os níveis da Fosfatase Alcalina resultou em níveis de 1369,36 UI/L, com o valor de referência sendo de $100 \mathrm{UI} / \mathrm{L}$. De acordo com Kruse et al., (2013) para cada aumento de $100 \%$ na Fosfatase Alcalina a probabilidade de morte aumenta 1,7 vezes, o que para este caso pode representar um fator de prognóstico desfavorável.

Ao final do processo cirúrgico, o nódulo removido foi enviado ao Setor de Patologia para exame histopatológico e resultou no tipo histológico osteossarcoma osteoblástico, sendo consistente com o que foi relatado por Kruse et al., (2013), que obtiveram $74 \%$ dos casos classificados como esse tipo, no entanto, ainda de acordo com os autores, o tipo histológico não influencia no tempo de sobrevida.

Após o retorno da paciente, observou-se a presença de um nódulo na raiz do membro pélvico esquerdo, sugestivo de metástase, posteriormente confirmado por exames ultrassonográfico e histopatológico. A ocorrência de metástase cutânea em casos de osteossarcoma é extremamente rara, pois a maior parte das metástases ocorrem nos pulmões, ossos e outros órgãos abdominais. Apesar da raridade, os achados deste relato podem ser comparados com os de Gorman et al., (2006). Adicionalmente, foi feito exame radiográfico que indicou a presença de nódulos de metástase pulmonar, o que corrobora com Lamb et al., (2019) que descreveram que em $26 \%$ dos casos de osteossarcoma desenvolvem-se múltiplos nódulos de metástase pulmonar.

\section{CONCLUSÃO}

Relatos de osteossarcoma em cães da raça Poodle não são muito comuns, assim como o desenvolvimento fora do esqueleto apendicular, destaca-se, ainda, a necessidade de maiores estudos epidemiológicos para avaliar o acometimento da raça com relação a neoplasia. No presente relato, evidencia-se a importância do exame citológico e histopatológico para um diagnóstico conclusivo. Ainda que a opção de tratamento quimioterápico tenha sido rejeitada pelo tutor, esta é, em combinação com o tratamento cirúrgico, uma das melhores formas para aumentar o tempo de sobrevida do paciente.

\section{REFERÊNCIAS}

BOERMAN, I.; SELVARAJAH G. T.; NIELEN M. and KIRPENSTEIJN, J. Prognostic factors in canine appendicular osteosarcoma - A meta-analysis. BioMed Central Veterinary Research (2012);8:56. Disponível em: <https://doi.org/10.1186/17466148-8-56>. doi: 10.1186/1746-6148-8-56 
CULP, W. T. N.; OLEA-POPELKA, F.; SEFTON, J.; ALDRIDGE, C. F.; WITHROW, S. J.; et al., ; Evaluation of outcome and prognostic factors for dogs living greater than one year after diagnosis of osteosarcoma: 90 Cases (1997-2008). Journal of the American Veterinary Medical Association. 245. 1141-6, 2014. Disponível em: <https://doi.org/10.2460/javma.245.10.1141 >. doi: 10.2460/javma.245.10.1141

FAN, T. M.; KHANNA C. Comparative Aspects of Osteosarcoma Pathogenesis in Humans and Dogs. Veterinary Sciences. 2(3):210-230, 2015. Disponível em: <https://doi.org/10.3390/vetsci2030210>. doi: 10.3390/vetsci2030210

FARCAS, N.; ARZI, B. and VERSTRAETE, F. J. (2014), Oral and maxillofacial osteosarcoma in dogs: a review. Veterinary and Comparative Oncology, 12: 169180, 2014. Disponível em: <https://doi.org/10.1111/j.1476-5829.2012.00352.x>. doi: 10.1111/j.1476-5829.2012.00352.x

FENGER, J. M.; LONDON, C. A.; KISSEBERTH, W. C. Canine Osteosarcoma: A Naturally Occurring Disease to Inform Pediatric Oncology, Institute for Laboratory Animal Research Journal, V.55, Issue 1, 1 January 2014, pg. 69-85, 2014. Disponível em: <https://doi.org/10.1093/ilar/ilu009>. doi: 10.1093/ilar/ilu009

GORMAN, E.; BARGER, A. M.; WYPIJ, J. M. and PINKERTON, M. E.; Cutaneous metastasis of primary appendicular osteosarcoma in a dog. Veterinary Clinical Pathology, 35: 358-361, 2006. Disponível em: <https://doi.org/10.1111/j.1939165X.2006.tb00149.x>. doi: 10.1111/j.1939-165X.2006.tb00149.x

KRUSE, M. A.; HOLMES, E. S.; BALKO, J. A.; FERNANDEZ, S.; BROWN, D. C. and GOLDSCHMIDT, M. H. Evaluation of Clinical and Histopathologic Prognostic Factors for Survival in Canine Osteosarcoma of the Extracranial Flat and Irregular Bones. Veterinary Pathology, 50(4), 704-708, 2013. Disponível em: <https://doi.org/10.1177/0300985812471542>. doi: 10.1177/0300985812471542

LAMB C. R.; WHITLOCK J; FOSTER-YEOW A. T. L. Prevalence of pulmonary nodules in dogs with malignant neoplasia as determined by CT. Veterinary Radiology \& Ultrasound. Pg.1-6, 2019.. Disponível em: <https://doi.org/10.1111/vru.12723>. doi: 10.1111/vru.12723

MONTINARO, V.; BOSTON, S. E.; BURACCO, P.; CULP, W. T. N.; ROMANELLI, G.; STRAW, R. and RYAN, S., Clinical outcome of 42 dogs with scapular tumors treated by scapulectomy: A Veterinary Society of Surgical Oncology (VSSO) retrospective study (1995-2010). Veterinary Surgery, 42: 943-950, 2013. Disponível em: <https://doi.org/10.1111/j.1532-950X.2013.12066.x>. doi: 10.1111/j.1532950X.2013.12066.x

SAPIERZYŃSKI, R. and CZOPOWICZ, M. ; The animal-dependent risk factors in canine osteosarcomas, Polish Journal of Veterinary Sciences, 20(2), 293-298, 2017. Disponível em: <https://doi.org/10.1515/pjvs-2017-0035>. doi: 10.1515/pjvs2017-0035 
SCHIFFMAN J. D.; BREEN M. 2015: Comparative oncology: what dogs and other species can teach us about humans with cancer. Phil. Trans. The Royal Society B370: 20140231. Disponível em: <http://dx.doi.org/10.1098/rstb.2014.0231>. doi: 10.1098/rstb.2014.0231

SIMPSON, S.; DUNNING, M. D.; BROT, S.; GRAU-ROMA, L.; MONGAN, N. P.; RUTLAND, C. S. Comparative review of human and canine osteosarcoma: morphology, epidemiology, prognosis, treatment and genetics, Acta Veterinaria Scandinavica pg. 59:71, 2017. Disponível em: <https://doi.org/10.1186/s13028-0170341-9>. doi: 10.1186/s13028-017-0341-9

SOTTNIK, J. L.; CAMPBELL, B.; MEHRA, R.; BEHBAHANI-NEJAD, O.; HALL, C. L.; KELLER, E. T. (2014): Osteocytes serve as a progenitor of osteosarcoma. Journal of Cellular Biochemistry, 115: pg. 1420-1429, 2014. Disponível em: <https://doi.org/10.1002/jcb.24793>. doi: 10.1002/jcb.24793

SZEWCZYK, M.; LECHOWSKI, R.; ZABIELSKA K. What do we know about canine osteosarcoma treatment? - review, K. Veterinary Research Communications, pg. 39: 61, 2015. Disponível em: <https://doi.org/10.1007/s11259-014-9623-0>. doi: 10.1007/s11259-014-9623-0 